\title{
A Preliminary Petri Net Model of the Transshipment Processes in the Taranto Container Terminal
}

\author{
Guido Maione \\ DIASS - Politecnico di Bari \\ Viale del Turismo, 8 \\ 74100 Taranto - Italy \\ gmaione@poliba.it
}

\author{
Michele Ottomanelli \\ DIASS - Politecnico di Bari \\ Viale del Turismo, 8 \\ 74100 Taranto - Italy \\ m.ottomanelli@poliba.it
}

\begin{abstract}
This paper is a contribution to define a complete and modular model of processes in container terminals, which can be used for simulation, test, monitor and control purposes. A schematic description of the main terminal processes is given, by specifying the resources handling containers in the Taranto Container Terminal. Then a Petri Net formalizes the sequence of operations, each involving the synchronization of resources, for the transshipment process, the efficiency of which seem to be the key to obtain good performances.
\end{abstract}

\section{Introduction}

Today efficiency of freight transportation depends on performance of intermodal transport supply system, which usually is a complex system made up of many different infrastructures and services. To achieve the best results, analysis, design, and control are necessary, possibly through flexible and adaptable modeling techniques and advanced simulation tools.

To this aim, the system is often represented as a set of modal networks (using railways, freeways, seaways, etc.), which intersect at particular intermodal nodes where different transport services are connected and goods change transport mode, e.g. at maritime container terminals. Each container used to transport goods is notoriously expressed in Twenty Equivalent Unit (TEU), to indicate a unit 20 feet long, 8 feet wide, and 8 feet high.

In a container terminal, it is important to guarantee fast operations to reduce delays in delivering goods to ships, trains and trucks, and consequently to reduce sea, road or railway transport time. Therefore, container terminals play a fundamental role in the interchange between road, railway and sea networks, then they are usually equipped with modern equipment, advanced transport systems and up-to-date information and communication technologies. In this context, the efficiency of a given terminal depends on its internal organization and management of the available facilities, according to planning and control strategies.

Container terminals typically provide many services all day long and seven days a week, e.g. container loading/unloading to/from vessel and feeder ships for import or export purposes, internal container movement from ships to stacking areas and vice versa, stacking containers in dedicated areas distributed in the terminal area, container inspection for customs requirements, reefer handling and storage, stuffing and unstuffing, etc.. Basically, the main processes which determine the system effectiveness are the following:

- unloading containers from a vessel or feeder ship to internal transfer vehicles, like trailers;

- moving containers from ship to stack;

- stacking containers in an assigned position;

- moving containers from stack to ship;

- loading containers from internal transfer vehicles to the vessel or feeder ship;

- consolidation, i.e. reorganization of the distribution of containers in the dedicated areas, to allow fast retrieval when necessary;

- transport of containers to other modes (railway, road). All the above processes need several shared and reusable resources and equipment, to fulfil the tasks involved in handling and transporting containers: quay cranes or yard cranes, transport vehicles, e.g. multitrailers or automatically guided vehicles (AGVs), straddle carriers, yard stacking deposits, automatic stacking cranes (ASC) or automatic storage/retrieval systems (ASRS), railway tracks, human operators. Quays, berths, yard and other specific areas can also be considered as space resources.

All processes and operations are usually planned, scheduled, monitored, and controlled by a central supervisor and make use of information technologies, to allow fast ship operations, optimization of the usage of facilities, and reduce lag times.

This simple and schematic overview makes evident the complexity of container terminals and the difficult job of describing, representing, monitoring and controlling the coexisting processes. To accurately solve this problem, no standard modeling or simulation tool 
exists even if several attempts have been made by scientific literature, based on mathematical models or empirical studies [2-8], Petri Nets (PN) formalism [1012] and discrete event simulation tools $[1,9]$.

In particular, to effectively support system operations design and control, it is commonly believed that simulation tools of the whole terminal are very helpful. Namely, managers and planners could use these tools to define the optimal system configuration and control strategies, in all the possible scenarios, and then to improve the performance even if difficult conditions occur (limited berthing space, congestion of internal traffic, few cranes or trailers available, etc.). Besides, a simulation tool is obviously useful to schedule, monitor and control in real-time all the operations performed by the terminal resources.

In this paper, we take the Taranto Container Terminal (TCT) as case-study [13]. Here, activities are growing and the terminal is planning to expand (e.g. quay and yard extensions, new equipment, increased TEUs capacity, infrastructure developments) because the commercial traffic is expected to grow in the near future from $36 \%$ to $45 \%$ of the total Mediterranean traffic share, due to the proximity to many markets. As far as the system is gaining complexity, infrastructures are developing, available resources are increasing, and the requirements for low costs and efficiency in the competition with other terminals are gaining more and more importance, the authors think that research can help in advising advanced models to represent, simulate, and on-line control the terminal activities.

So, this paper is a first attempt to define a complete model for the processes in a container terminal, which could be useful to define a simulation platform to test different system configurations, equipment organizations, and control strategies, eventually with reference to literature benchmarks. In particular, the case of the TCT is considered and digraphs and PN are used to model the main processes due to their power in graphically representing synchronized and parallelized activities executed by means of shared resources.

The paper is organized as follows. Section 2 briefly reviews some scientific contributions on modeling and solving problems in container terminals. Section 3 presents the system under investigation, by specifying the resources involved and the main processes. Section 4 gives a PN model of the transshipment fundamental process. Section 5 draws the conclusion.

\section{Some literature review}

In a dynamic and complex container terminal many and different operations have to be carried out to supply the requested services. Thus, it is important to define the most convenient way to model such a system with respect to the design, analysis, monitoring, and control aims.
Different approaches have been proposed to model operations depending on the planning level (i.e., strategic, tactical, operational).

Many works have been focused on modeling single operations, sequence of operations or the complete system. Anyway, each single problem has to be systematically considered as an integrated part of the whole in order to improve the service supply chain.

Apart from problems at strategic level, such as location of the terminal, number of cranes, number of berths, etc., many works have been focused on the main operational problems, such as:

- Allocation of ships to available berths;

- Loading and unloading of the ships;

- Transfer of containers from ships to storage area and vice versa;

- Stacking of containers;

- Transfer to/from other modes (road, rail, water);

- System simulation.

The problem of containers transfer from ship to stack and vice-versa is discussed by many authors considering both full-automated and not automated transfer systems [1]. The allocation of ships to berths consist in assigning incoming ships to berthing positions in order to minimize the waiting time of the vessel. Problem solutions have been discussed in [2-3]. The ships loading/unloading operations cover many problems, for example the quay cranes assignment [7], the unloading plan [4-5], the stowage plan [6].

Simulating the whole system suffers complexity since many problems are linked by shared resources whose availability is time dependant. Nevertheless, efficiency of terminal management can be achieved by means of system simulation. Namely, it is possible to design and evaluate control strategies, different system layouts, new and powerful technologies and processes. To this aim, a resources allocation tool is proposed in [8] where the model is based on operations research methods and allows managers to develop resources allocation plans. A distributed discrete-event container terminal simulator based on object-oriented modeling technique was also recently proposed in [9]. A comprehensive review of terminal containers simulation models is given in [1].

PN represent a well-established modeling tool. Their basic capability is in the graphical representation and formal analysis of all processes in a discrete event system [14]. PN are suitable to describe precedence relations, synchronization, mutual exclusion and many other forms of interaction between the concurrent events. When compared with other existing formalisms, PN show the possibility to derive formal properties, which allow safe and efficient operation. So, they allow to identify critical system conditions (blocking, deadlock, congestion) by PN figures, f.e. empty siphons for deadlocks. This can help the system designer in preventing these states, which can critically reduce the terminal performance. PN have been used in [10-12] to 
model and analyze container terminals with respect to different aspects of terminal operations. In [10] a stochastic PN models containers transfer in a rail-road transshipment yard. In [11-12], PN describe the synchronization problems in using shared resources, and the analysis of faulty situations.

\section{System description}

Here, we consider the TCT, a site managed by a private service company providing container handling services [13]. The terminal is located at Taranto, in the south-east ionic area of Italy, facing the Mediterranean region in a strategic geographical location. Namely, it is situated along the Suez-Gibraltar shipping route and serves both Mediterranean, Adriatic and Black Sea ports and beyond destinations. Connections to road and rail networks exist to get access to regional, national and European markets. The ships TCT can receive range from feeders to the last generation of $6,000+$ TEU giant carriers. Taranto is ranked on the third position among the Italian industrial and commercial ports, in terms of handled tons and containers. The TCT management currently reports good performances like throughput trend: a total movement of about 61,829 TEUs/month, of which 6,214 TEUs/month are imported by road or railway systems, and $4,745 \mathrm{TEUs} / \mathrm{month}$ are imported only by railways (data updated to March '04).

\subsection{Terminal Resources}

The site is organized in distinct space resources (see figure 1). Basically, the processes occur in an overall area of $930,000 \mathrm{~m}^{2}$, in which full or dangerous containers are stored in a yard of $250,000 \mathrm{~m}^{2}$, in 7,062 slots for a total capacity of 35,310 TEUs, and empty containers are stored in a space of $40,000 \mathrm{~m}^{2}$ with 45,000 TEUs capacity). We may distinguish:

- a quay where ships are allocated berths;

- an area for stacking full containers, which is virtually divided into blocks, numbered from 11 to 46; some blocks (from 11 to 26) are for export containers, some other for import containers (from 32 to 46); a reefer containers yard is allocated in blocks 44 and 45 ;

- areas (from M1 to M12) for empty containers, including a block (M4) for scanning;

- an area for dangerous containers (DG);

- a stripping area (STRIP), a customs area (EX), a parking area for primary transport vehicles (PARK), a washing and maintenance area (W\&M), a fuel station (FU), a railway connection ( 5 tracks $1,000 \mathrm{~m}$ long each), the operations control centre (CC), and, finally, a gate $(G)$ for trucks to enter/exit the terminal.

Actually, there is only one quay with a capacity of 3 ships ( 5 virtual berths), for a total length of $1,500 \mathrm{~m}$. Ship allocation to available berths is not a significant problem since ship arrivals are planned such that no more than $2 / 3$ ships are contemporaneously served. The quay is equipped with: 6 one-way lanes to route the internal transport vehicles; 10 quayside gantry cranes (QC), called portainers, of which 2 are Ultra Post Panamax (55/65 tons lifting capacity) and 8 are Super Post Panamax (45/55 tons lifting capacity). These cranes are able to load/unload containers onto/from the ship.

The yard is equipped with: 22 rail mounted yard gantry cranes (YC), called yard transtainers, which may load/unload containers onto/from transport vehicles (40 tons lifting capacity). Namely, $7 \mathrm{YC}$ serve the blocks in the first row (DG,11,12,13,14,15,16), 6 YC serve the second row (DG,22,23,24,25,26), $5 \mathrm{YC}$ the third row $(32,33,34,35,36), 4 \mathrm{YC}$ the fourth row $(43,44,45,46)$.

As for the rubber-tyred equipment, there is 1 jolly mobile harbour crane (HC), 3 reach stackers (RS) for activities in DG and EX areas and for positioning containers from trailers to trucks (40 tons lifting capacity), 5 side loaders (SL) for handling empty containers, 50 trailers (TR). To establish the connection to the rail network, 2 railway cranes (RC), called transtainers (40 tons lifting capacity), load/unload containers onto/from railway trucks.

Last but not least, skilled workforce and information and communication technologies are used for terminal management and real-time container tracking. For sake of simplicity, human workforce is neglected in the model even if it is a system resource.

All resources can be classified as dedicated or shared resources. Resources dedicated to specific areas (QC, $\mathrm{YC}, \mathrm{RC}$ ) belong to the first class, while other resources (TR, HC, RS, SL) are shared as they can move through different terminal areas.

\subsection{Terminal processes}

TCT is a node in which three modes of transportation are possible: sea, railway, and road. Containers may follow three handling cycles (table I): export, when they arrive through railway/road, by means of trains/trucks, and depart on a vessel ship; import, when they arrive with a ship (vessel/feeder) and depart by trains/trucks; transshipment, when arrival and departure are by ships. In the last case, a big vessel ship arrives after a long distance trip to leave containers, which are then loaded on a small feeder ship departing for a short trip; or a feeder arrives to leave containers for a vessel for a far away destination. Most of the maritime traffic currently handled by TCT (about 90\%) is transshipment.

\begin{tabular}{|c|c|c|}
\hline Cycle & Arrival Mode & Departure Mode \\
\hline Exp Road & Truck & Ship \\
\hline Exp Railway & Train & Ship \\
\hline Imp Road & Ship & Truck \\
\hline Imp Railway & Ship & Train \\
\hline Transshipment & Ship (vess./feed.) & Ship (feed./vess.) \\
\hline
\end{tabular}

Table I. Operating cycles at TCT. 


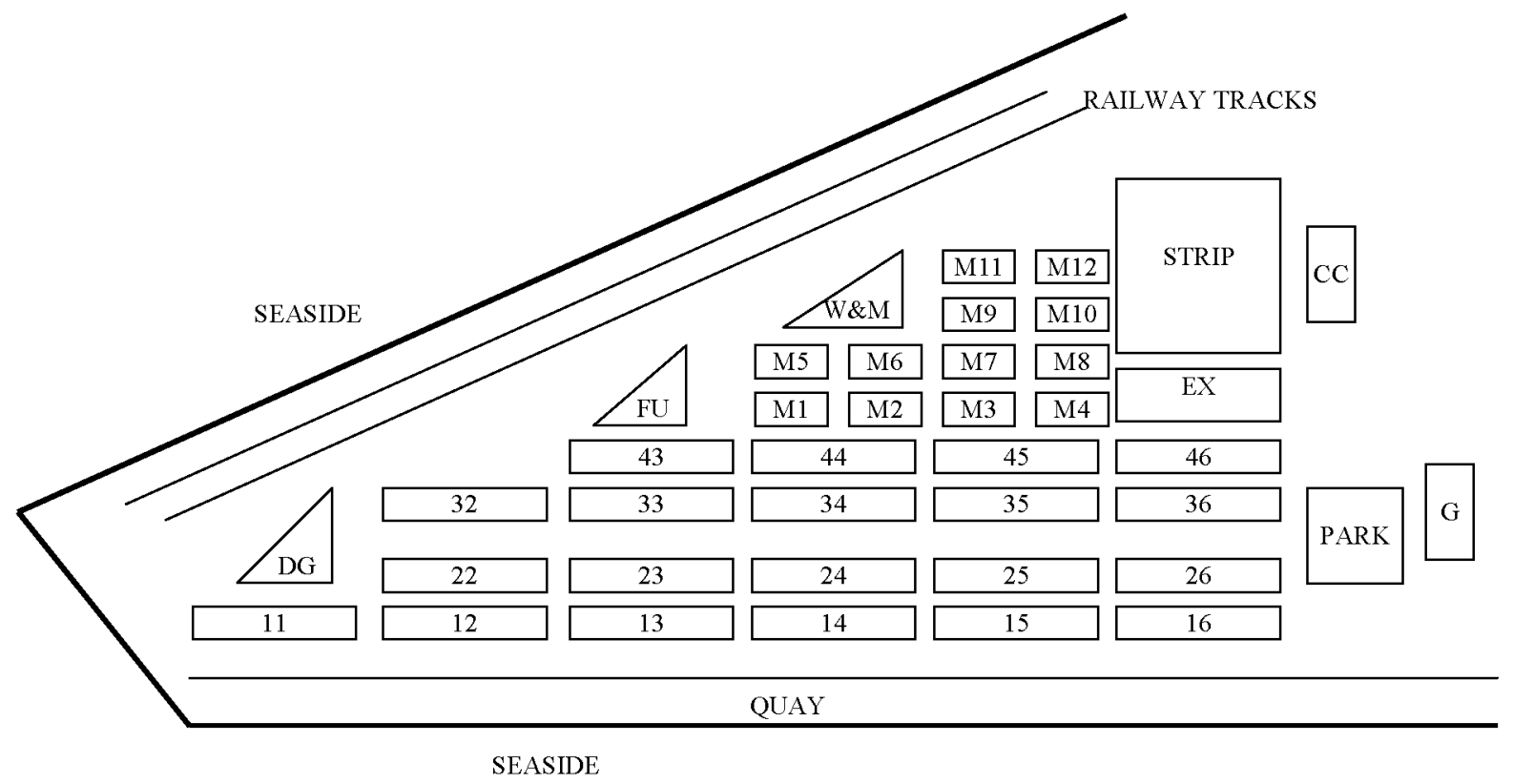

Figure 1. Taranto Container Terminal: schematic view.

All cycles followed by containers require a sequence of operations, which make use of the terminal resources and can be graphically represented by directed graphs as in figures 2, 3, and 4. Export/import cycles involving road transportation are depicted in figure 2 , export/import cycles involving railway in figure 3, transshipment in figure 4 .

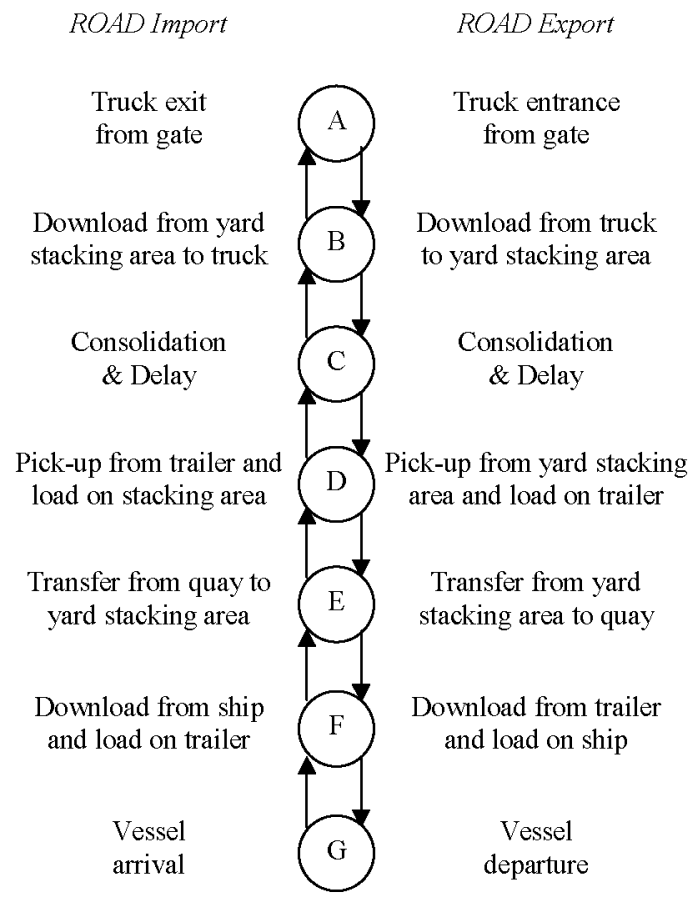

Figure 2. Road export/import cycles.

In figures 2 and 3 , top-down arrows indicate the export sequence, bottom-up arrows the import sequence.
RAIL. Import

RAIL. Export

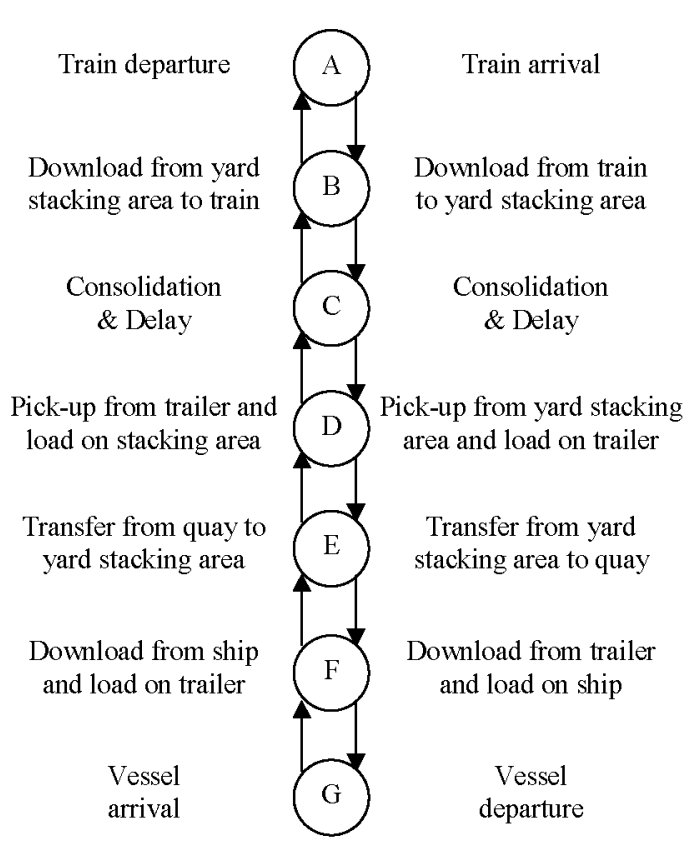

Figure 3. Railway export/import cycles.

Each node models an operation executed on containers and can be represented by a PN module. Arcs represent the precedence relations between successive operations. All operations have a certain duration, depending on the hardware devices. If consolidation occurs, its duration is unpredictable, but when it is not necessary containers wait in their stacked position for a certain delay time (10 days on the average). 
Tables II, III, and IV indicate the required resources for each operation in the road and railway import/export and in the transshipment cycles, respectively.

\begin{tabular}{|c|l|}
\hline Operation & Resource \\
\hline A & Gate \\
\hline B & RS \& TR \& YC \\
\hline C, D & TR \& YC \\
\hline E & TR \\
\hline F & TR \& QC \\
\hline G & Quay \& berth \\
\hline
\end{tabular}

Table II. Resources required in road import/export cycle.

\begin{tabular}{|c|l|}
\hline Operation & Resource \\
\hline A & Railway trucks \\
\hline B & RC \& TR \& YC \\
\hline C, D & TR \& YC \\
\hline E & TR \\
\hline F & TR \& QC \\
\hline G & Quay \& berth \\
\hline
\end{tabular}

\section{Table III. Resources required in railway} import/export cycle.

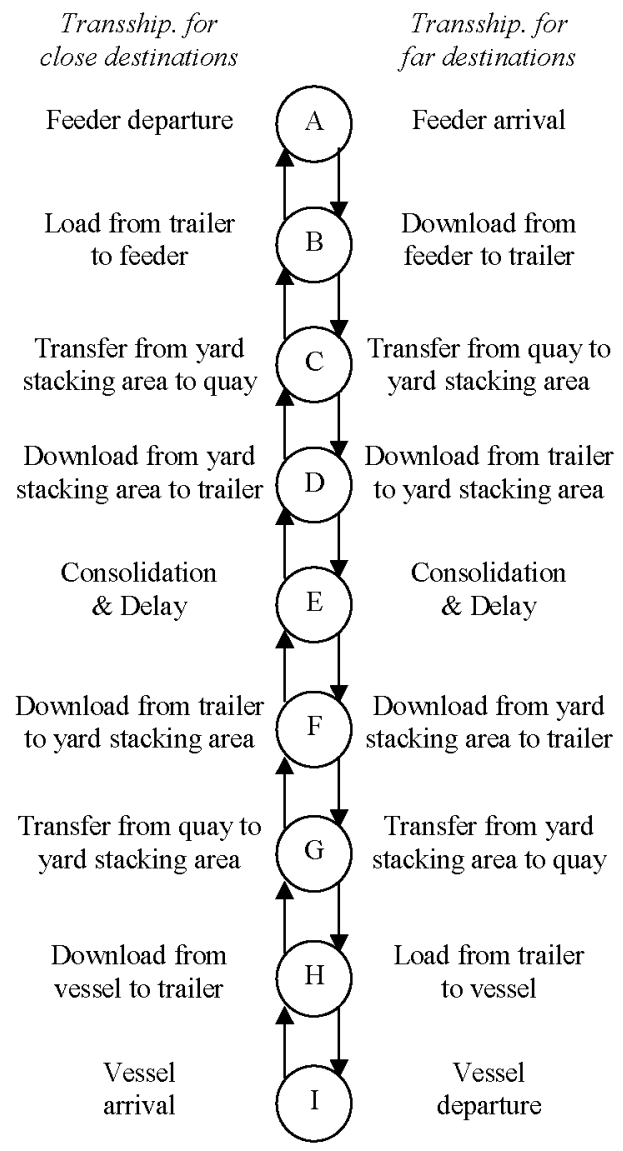

Figure 4. Transshipment cycle.

\begin{tabular}{|c|l|}
\hline Operation & Resource \\
\hline A, I & Quay \& berth \\
\hline B, H & QC \& TR \\
\hline C, G & TR \\
\hline D, E, F & TR \& YC \\
\hline
\end{tabular}

\section{Table IV. Resources required in transshipment cycle.}

As schematically and synthetically described, the main yard and quay activities executed by the terminal resources, we will focus on, are: unloading and loading of a ship, internal transfer from ship to stack and viceversa, stacking, transfer to other modes.

To guarantee accuracy and effectiveness in modelling and analysis, discrete-event dynamic systems seem the more appropriate formalism to represent the complex terminal system. In particular, Petri Nets give an immediate and powerful graphical representation of all the necessary operations, the shared resources, the synchronized or parallelized process, etc. [15-16]. They can also be used to foresee, detect and prevent particular problems, e.g. the likelihood of system congestion, blocking phenomena, and deadlocks. To this aim, most scientific literature use PN, even if other digraphs seem more efficient to simplify models and detect particular system states when the model is complex [17].

\section{Petri Net model of transshipment operation cycle}

\subsection{Basic Petri Nets notations}

We briefly recall just the basic notations on PN that we use for modeling purpose.

An ordinary $\mathrm{PN}$ is a bipartite graph formalized by a four-tuple $\mathbf{N}=\left(P, T, A, M_{0}\right)$, in which places in set $P$ and transitions in set $T$ are linked by $\operatorname{arcs}$ in set $A$ to represent how the system state changes, as tokens, representing resource-units or entities (i.e. containers) in process, flow through the net.

More specifically, places are of two different types: the first one models the execution of activities, the second one the availability of resources (cranes, vehicles, etc.). Each place represents a condition and is depicted by a circle. Each resource $r_{i}$ has a finite capacity $C\left(r_{i}\right)$ (number of units) to serve containers.

Transitions are graphically shown with bars and model events changing the state. Each state value is defined by the marking vector $M: P \rightarrow\{0,1,2, \ldots\}$, where $M\left(p_{i}\right)$ gives the current number of tokens in $p_{i} \in \mathrm{P}$. An unmarked place doesn't contain tokens and a marked place contains as many tokens as $\mathrm{M}\left(p_{i}\right) . M_{0}$ : $P \rightarrow\{0,1,2, \ldots\}$ is the initial marking. Tokens are black dots put inside places. 
Input arcs are oriented from places to transitions, and output arcs from transitions to places, and all are drawn as arrows. They show us how places and transitions are connected. Namely, a transition $t$ has a certain number of input (and output) places, which are the pre-conditions (post-conditions) associated with $t$. The pre-conditions enable transition $t$, while the post-conditions are defined after the occurrence of the event modeled by $t$.

So, the PN states (or markings) do change according to the following basic rules:

a) enabling rule: a transition is enabled if each of its input places is marked, but an enabled transition may or may not fire (depending on whether or not the event actually takes place);

b) firing rule: a firing of an enabled transition consumes a token from each input place and produces a token for each output place. We assume that only one transition fires at a time.

We consider the operation cycles followed by containers (see figures 2, 3 and 4) in the same way as working procedures in manufacturing systems. Then each cycle can be specified by a sequence of operations, performed by the terminal resources that are successively acquired by each container which is served by the system. The flow of containers through the system resources is ruled by the asynchronous occurrence of discrete events, which determines the evolution of the state of the system modeling the container terminal. Typically, the state variables are only permitted to jump at discrete points in time from one discrete value to another.

\subsection{Petri Net of the transshipment cycle}

Now we focus on the transshipment cycle (figure 4) to derive the corresponding PN. The same procedure can be followed for export and import cycles, which is omitted here for sake of brevity.

On the basis of the description previously made (see figure 4 and table IV), we may build the $\mathrm{PN}$ in figure 5 , made of places and transitions as described in table $\mathrm{V}$. For sake of space and simplicity, the net is split in two successive parts, linked by $t_{6}$, and identical places and transitions (i.e. $\mathrm{t}_{6}, \mathrm{r}_{1}, \mathrm{r}_{2}, \mathrm{r}_{3}$ ) are replicated but represent the same condition or event.

The displayed PN represent the initial state after a ship arrival: the dot in place $p_{0}$ represents the number of containers (tokens) available for unloading by the considered QC; the dot in $r_{1}$ represents the unique QC; the tokens in $r_{2}$ are the number of TR available for transporting containers (50 in total, maybe less for the adopted unloading/loading plans); the tokens in $r_{3}$ are the number of YC available for stacking containers (depends on the served row, see section 3.1).

All timed transitions represent processes whose duration can be a random variable, so that we may have a Generalized Stochastic Petri Net, which may be useful to perform statistical analysis.
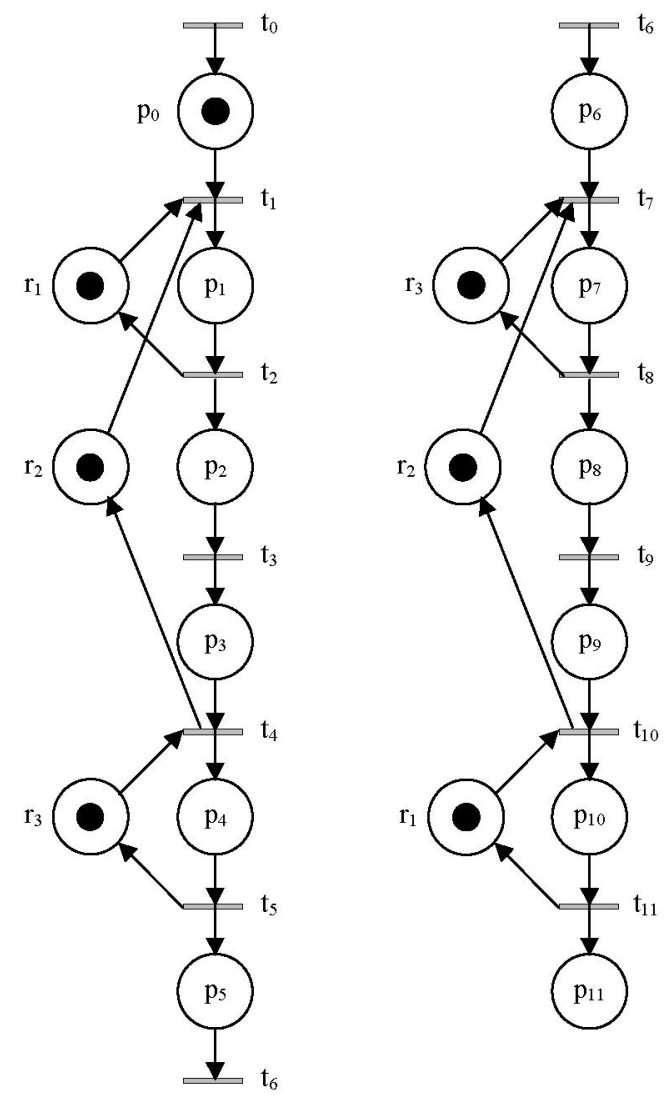

Figure 5. Petri Net of the transshipment cycle.

The only peculiarity is the necessity of resources $r_{2}$ and $r_{3}$ (TR and YC) in case consolidation occurs, which would require the proper connection of the associated places to transition $t_{6}$, which is not shown for figure clarity.

\begin{tabular}{|c|c|c|c|}
\hline$P$ & Meaning & $T$ & Meaning \\
\hline $\mathrm{p}_{0}$ & $\mathrm{C}$ available on ship & $\mathrm{t}_{0}$ & Ship arrival \\
\hline $\mathrm{p}_{1}$ & C picked-up by QC & $\mathrm{t}_{1}$ & QC picking C from ship \\
\hline $\mathrm{p}_{2}$ & $\mathrm{C}$ on $\mathrm{TR}$ & $\mathrm{t}_{2}$ & QC dropping $\mathrm{C}$ on TR \\
\hline $\mathrm{p}_{3}$ & $\mathrm{C}$ on TR close to block & $\mathrm{t}_{3}$ & TR transferring $\mathrm{C}$ to yard \\
\hline $\mathrm{p}_{4}$ & C picked-up by $Y C$ & $\mathrm{t}_{4}$ & YC picking $\mathrm{C}$ from $\mathrm{TR}$ \\
\hline $\mathrm{p}_{5}$ & C stacked in block & $\mathrm{t}_{5}$ & YC stacking $\mathrm{C}$ in block \\
\hline $\mathrm{p}_{6}$ & $\mathrm{C}$ available for transf. to ship & $\mathrm{t}_{6}$ & Consolidation \& Delay \\
\hline $\mathrm{p}_{7}$ & C picked-up by $Y C$ & $\mathrm{t}_{7}$ & YC picking C from block \\
\hline $\mathrm{p}_{8}$ & $\mathrm{C}$ on $\mathrm{TR}$ & $\mathrm{t}_{8}$ & YC dropping $\mathrm{C}$ on TR \\
\hline $\mathrm{p}_{9}$ & $\mathrm{C}$ in quay area & $\mathrm{t}_{9}$ & TR transferring C to ship \\
\hline $\mathrm{p}_{10}$ & C picked-up by QC & $t_{10}$ & QC picking C from TR \\
\hline $\mathrm{p}_{11}$ & C loaded on ship & $\mathrm{t}_{11}$ & QC loading C on ship \\
\hline $\mathrm{r}_{1}$ & QC availability & & \multirow{3}{*}{$\mathrm{C}$ stands for container } \\
\hline $\mathrm{r}_{2}$ & TR availability & & \\
\hline$r_{3}$ & YC availability & & \\
\hline
\end{tabular}

\section{Table V. Places and transitions in PN.}

According to the PN notations, we may note that even simple processes can give rise to complex PN, defined by a lot of places, transitions and arcs. Typically, the 
number of PN states is combinatorial exploding. Then, both the PN and the algorithms for state exploration in these models are not easy to be implemented.

This sometimes motivates more efficient models. In particular, digraph models are simpler than $\mathrm{PN}$, even if less common. Their strong point is in the easy system representation and in the efficiency of algorithms for identification of deadlocks. In fact, the number of vertices and of edges in a digraph $D=(V, E)$ is much lower than in PN. The vertices in $V$ are system resources while each edge in $\mathrm{E}$ represents a precedence relation between two adjacent resources in an operation sequence. For each operation, a part acquires and uses a single unit of resource, and releases it at the end of process only if it acquires the next resource.

Then, if resources are limited and the number of sequences increases, the vertices in $V$ remain fixed while places in $P$ and transitions in $T$ augment. Besides, the edges in $E$ grow less than arcs in $A$. So, the number of nodes and links in a PN is much higher than in $D$.

In this paper, we choose PN to obtain a complete, detailed model of the system. Eventually digraphs are more efficient from a computational stand-point. Also, the PN model can be implemented in a simulation model to monitor the system and measure its efficiency in the transshipment or other processes, in terms of standard TEUs throughput indices. Preliminary simulations were performed on the basis of the PN description of processes. Simulation results confirmed the PN modeling power, because they were in good accordance with real system performance. In particular, simulation confirmed the real TEUs movement registered in 2004 .

\section{Conclusion}

In this paper, we presented a first PN model of the transshipment processes occurring in the Taranto Container Terminal site. The model is modular and can be easily expanded to take other processes into account. Also, it can be used to develop a simulation platform to measure system performance indices and test control policies, eventually using corresponding simplified digraph models to detect and prevent dangerous states.

\section{Acknowledgement}

The authors thank Eng. Fornaro of TCT for his kind assistance and Miss Pizzileo, student of Politecnico di Bari at Taranto, for her big contribution.

\section{References}

[1] I.F.A. Vis and R. De Koster, "Transshipment of containers at a container terminal: An overview", Europ. Jour. of Operational Research, Vol. 147, pp. 1-16, 2003.
[2] J.-F. Cordeau , G. Laporte, P. Legato and L. Moccia, "The Berth Allocation Problem: Application to the Gioia Tauro Marittime Terminal", Technical Report no. 17. C.R.T., University of Montreal, Canada. 2003.

[3] A. Imai, E. Nishimura and S. Papadimitriou, "The Dynamic Berth Allocation Problem for a container Port", Transportation Research, Vol. 35B, pp. 401-417, 2001.

[4] K.H. Kim, Y.M. Park and K.-R. Ryu, "Deriving decisions rules to locate export containers in container yards", Eur. Jour. of Oper. Res., Vol. 124, No. 1, pp. 89$101,2000$.

[5] G. Crainic, M. Gendreau and P. Dejax, "Dynamic and stochastic models for the allocation of empty containers", Operations Research, Vol. 41, pp. 102-126, 1993.

[6] D. Ambrosino, A. Sciomachen and E. Tanfani, "Stowing a containership: the master bay plan problem", Transportation Research, Vol. 38A, pp. 81-99, 2004.

[7] R.I. Peterkofsky and C.F. Daganzo, "A branch and bound solution method for the crane scheduling problem", Trans. Res., Vol.24B, No.3, pp.159-172, 1990.

[8] L.M. Gambardella, A.E. Rizzoli and M. Zaffalon, "Simulation and planning of an intermodal container terminal", Simulation, Vol. 71, No. 2, pp. 107-116. 1998.

[9] M. Bielli, A. Boulmakoul and M. Rida, "Object Oriented Model For Container Terminal Distributed Simulation", to appear on European Journal of Operational Research.

[10] M. Fischer and P. Kemper, "Modeling and Analysis of a Freight Terminal with Stochastic Petri Nets", Proc. of 9th IFAC Int. Symp. Control in Transp. Systems, Vol. 2, pp. 195-200, Braunschweig, Germany, June 2000.

[11] C. Degano and A. Di Febbraro, "On using Petri nets to detect and recover from faulty behaviours in transportation facilities", Proc. IEEE Int. Conf. Sys., Man and Cybe., Yasmine-Hammamet, Tunisia, 6-9 Oct. 2002.

[12] C. Degano and A. Di Febbraro, "Modelling Automated Material Handling in Intermodal Terminals", Proc. IEEE/ASME Intern. Conf. on Advanced Intelligent Mechatronics, Como, Italy, 8-12 July 2001.

[13] Taranto Container Terminal, official web-site, http://www.tct-it.com/index.html.

[14] C.G. Cassandras, S. Lafortune, Introduction to discrete event systems, Kluwer, 1999.

[15] T. Murata, "Petri nets: properties, analysis, and applications", Proceedings of the IEEE, Vol. 77, No. 4, pp. 541-580, April 1989.

[16] J.L. Peterson, Petri Net Theory and the Modeling of Systems, Prentice-Hall, Englewood Cliffs, NJ, USA, 1981.

[17] G. Maione and D. Naso, "New control policies preventing deadlock in automated manufacturing systems", Proc. 9th IEEE Int. Conf. on Emerging Technologies and Factory Automation (ETFA '03), Lisbon, Portugal, Vol. 2, pp. 81- 86, 16-19 Sept. 2003. 